\title{
EXTENDED SEQUENCE MODELLING IN DESIGN ENGINEERING - GAINING AND DOCUMENTING KNOWLEDGE ABOUT EMBODIMENT FUNCTION RELATIONS WITH THE C\& C $^{2}$-APPROACH
}

\author{
Matthiesen, Sven; Grauberger, Patric; Schrempp, Lukas \\ Karlsruhe Institute of Technology (KIT), IPEK - Institute of Product Engineering
}

\begin{abstract}
In embodiment design, functions are implemented in a technical systems embodiment. For doing so, design engineers need to understand the relations of embodiment and function. Many systems change their states during function fulfilment which complicates their relations and leads to ambiguity in design decisions. The challenge for design engineers is that they often need to make important decisions about the design before they can use sophisticated analytical models to investigate them. This contribution presents a structure for the $\mathrm{C} \& \mathrm{C}^{2}$-Sequence Model as a non-analytical model to support design engineers in modelling embodiment function relations. This structure contains four dimensions that are derived from the state of the art and preliminary work. It enables the structuring of gained knowledge about embodiment function relations and supports their communication in design engineering teams. Two development projects in academic and corporate environment are conducted using the structure to investigate its applicability. In these projects, design engineers were able to document and use gained knowledge about the investigated complicated systems.
\end{abstract}

Keywords: Embodiment design, Product modelling / models, Design engineering, Design methods, Early design phases

\section{Contact:}

Grauberger, Patric

Karlsruhe Institute of Technology

IPEK - Institute of Product Engineering

Germany

patric.grauberger@kit.edu

Cite this article: Matthiesen, S., Grauberger, P., Schrempp, L. (2019) 'Extended Sequence Modelling in Design Engineering - Gaining and Documenting Knowledge about Embodiment Function Relations with the C\&C2-Approach', in Proceedings of the 22nd International Conference on Engineering Design (ICED19), Delft, The Netherlands, 5-8 August 2019. DOI:10.1017/dsi.2019.154 


\section{INTRODUCTION}

In embodiment design, a technical system is defined which can fulfil its functions under consideration of requirements and constraints. Design engineers need to define the systems embodiment based on their knowledge about the relations of design parameters (details of the embodiment) and functions (including system behaviour). These relations are described as embodiment function relations (EFRs) in the following. This is challenging, as often only the embodiment is documented (e.g. in technical drawings) and design engineers need to build a mental model of the EFRs based on this. In addition, requirements and constraints are often changed or extended in the course of a development project (Morkos et al., 2012). This is due to the co-evolution of problem and solution (Dorst and Cross, 2001). In order to be able to react to this, the knowledge of EFRs is crucial for design engineers.

Some of the EFRs are well investigated and can be changed using tables or calculations, for example the relation of torque transmission in a gear pair with the cylindrical press fitting. Other EFRs are not standardized and therefore more difficult to understand. They are first thought through in mental models of the design engineers (Matthiesen, 2011). Explicit models can be derived from them if required. In today's mechatronic systems, EFRs in their mechanical parts can become very complicated and explicit models are usually indispensable for analysis.

These explicit models can be differentiated into executable analytical models, such as FEM or multibody simulation models, and non-executable models, such as the Characteristics Properties Model (CPM) (Weber, 2014), models based on the Function-Behaviour-Structure Framework (Gero and Kannengiesser, 2004), the "Organ Domain" models by (Andreasen et al., 2015) or the Contact and

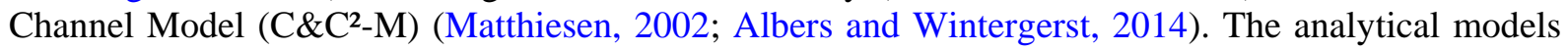
are usually not available until a parameterized product or subsystem is present, as they need it as basis. They can be used for the analysis and synthesis of the product to be designed once the system has reached the necessary level of maturity.

According to the "Rule of 10", necessary changes to the system become more and more elaborate as development progresses (Ehrlenspiel and Meerkamm, 2017). Therefore, it is important to make expedient design decisions as early as possible. Gaining the necessary knowledge about EFRs depends mostly on the experience of the design engineers. They often use visualizations of the system to support their thinking processes in the embodiment design phase. From the non-executable models, the $\mathrm{C} \& \mathrm{C}^{2}$-Approach and the Organ Domain models contain a visualization of the systems embodiment.

A special challenge in gain of knowledge about EFRs occurs when the system changes its state during function fulfillment. This often leads to changing, emerging and disbanding EFRs and a complicated system behaviour. Complicated means here, that EFRs can still be identified, however the necessary effort increases dramatically. These state changes are taken into account in the analytical models but their modelling is hardly supported in non-executable models. The SysML sequence diagrams (Weilkiens, 2007) consider state changes of technical systems, however it focusses on modelling of requirements and functional interrelations. Therefore it doesn't include a visualization of the systems embodiment and elements to depict EFRs and can't support design engineers in explicating their mental models of EFRs.

A model that currently supports the modelling of EFRs in systems that change their states is the $\mathrm{C} \& \mathrm{C}^{2}$-Sequence Model (Albers et al., 2008). It is based on the $\mathrm{C} \& \mathrm{C}^{2}$-Approach and shows potential for modelling complicated EFRs, however it has limited applicability due to structural limitations. Created models become incomprehensible and are no longer helpful, when EFRs have to be considered on different levels of detail in different states of the system which is often the case in complicated systems.

The challenge arising from this is, that no non-executable model for EFRs in complicated systems is present for support of design engineers in early phases of embodiment design. From this challenge, the research question of this contribution is derived:

Which structure needs a model for embodiment function relations in order to support design engineers in gain of knowledge about complicated systems?

This contribution describes the development of a structure for the $\mathrm{C} \& \mathrm{C}^{2}$ sequence model, which supports explicit expression of the mental models of design engineers in analysis of complicated systems. From the 
state of the art in modelling of EFRs, dimensions are identified which are important in order to be able to document gained insights in complicated systems. Then, the $\mathrm{C} \& \mathrm{C}^{2}$-Sequence Model is extended through a

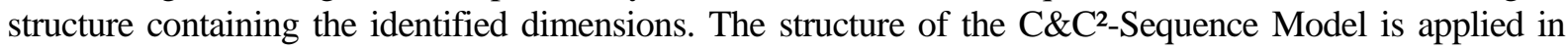
two development projects in embodiment design, where analyses of complicated systems are conducted.

\section{METHODS FOR MODELLING EMBODIMENT FUNCTION RELATIONS IN COMPLICATED SYSTEM}

The developed structure uses the $\mathrm{C} \& \mathrm{C}^{2}$-Sequence Model, which to some extend already covers the analysis of complicated systems. This model is based on the $\mathrm{C} \& \mathrm{C}^{2}$-Approach as a modelling approach for EFRs that uses visualization of the embodiment of a technical system. It also uses preliminary work for modelling state changes in technical systems. The $\mathrm{C} \& \mathrm{C}^{2}$-Approach and preliminary work on modelling state changes are briefly explained in the following.

\subsection{The contact and channel approach (C\&C $\mathbf{C}^{2}$-Approach)}

The $C \& C^{2}$-Approach is a thinking tool for embodiment design. Its aim is to support design engineers in recognizing function relevant parameters of the embodiment. As a meta-model it contains elements and rules to build up explicit models and can be seen as a language that provides words for explicit documents. It consists of three key elements and three basic hypotheses that define the usage of its key elements. Its key elements are the Working Surface Pair (WSP), Channel and Support Structure (CSS) and the Connector (C). (Matthiesen, 2002)

A WSP describes the interface, where parts of the system connect while it fulfills its function. The CSS runs through system parts and connects the WSP. A CSS can include parts of components or whole subsystems, according to the modeling purpose. The $\mathrm{C}$ sets the system boundary and transfers influences from outside the boundary into the system (Albers and Wintergerst, 2014). These elements are depicted in Figure 1 on the left side. They contain parameters of the embodiment that are relevant for the function fulfillment. For example a friction coefficient is a parameter of a WSP, the stiffness of a component or subsystem is a parameter of a CSS. These parameters cause the systems behaviour and are implemented or adjusted by design engineers in embodiment design. The $\mathrm{C} \& \mathrm{C}^{2}$-Approach supports documentation of these parameters and their relation to functions in the system. The basic hypotheses describe possibilities and boundaries of modeling with the $\mathrm{C} \& \mathrm{C}^{2}$-Approach (Matthiesen, 2002). They are depicted in Figure 1 (right side). The first basic hypothesis states, that function needs interrelations of components through WSP. The second basic hypothesis states that a function is fulfilled through a minimum of two WSPs that are connected by a CSS and integrated in the environment by Connectors. The third basic hypothesis describes the fractal character of modelling and shows, how the created $\mathrm{C} \& \mathrm{C}^{2}$-Models of a system differs according to point of view and purpose of modelling. From the key elements and basic hypotheses, a $C \& C^{2}-M o d e l$ is created (Figure 1, centre). (Matthiesen et al., 2018)

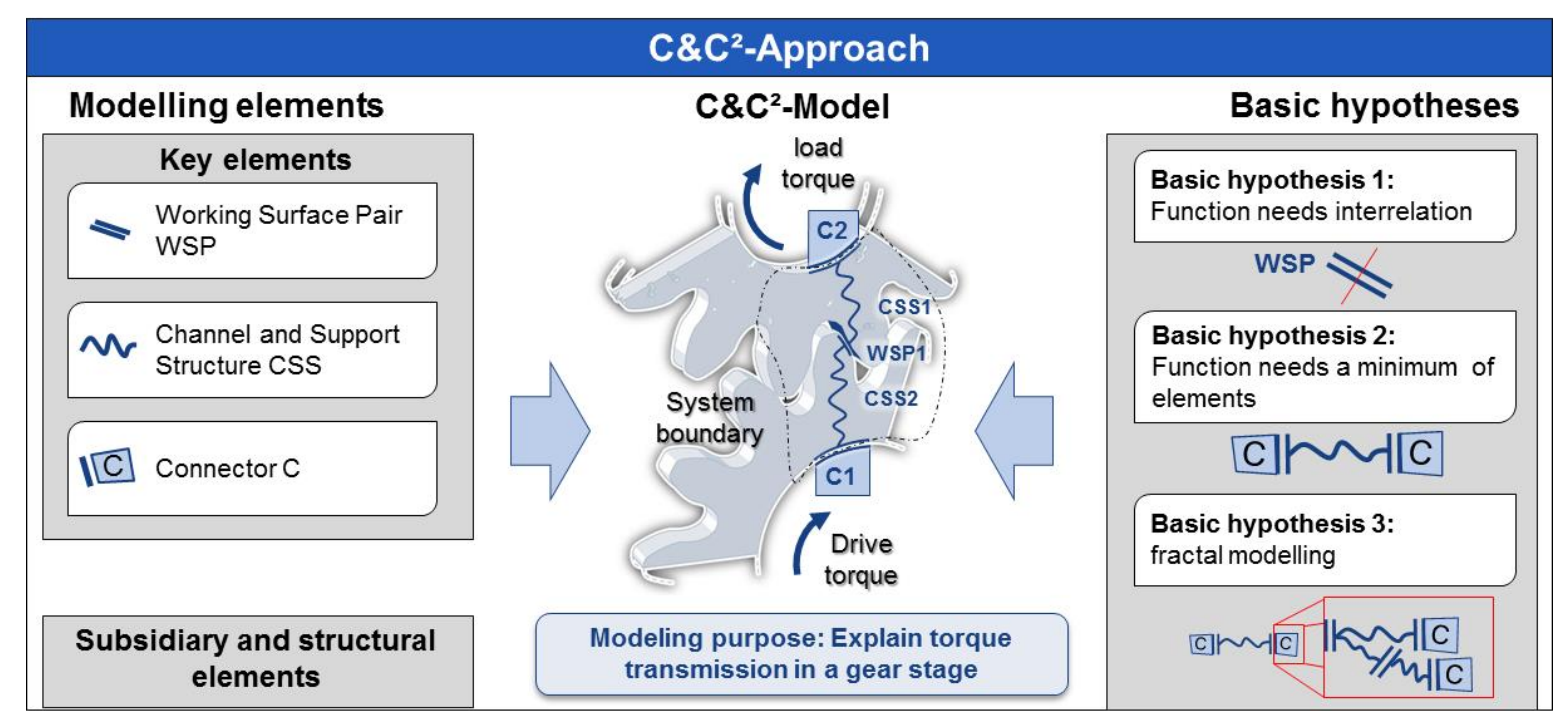

Figure 1: Overview of the C\&C2-Approach and its elements according to (Matthiesen et al., 2018) 


\subsection{C\&C² Sequence Model}

With the $C \& C^{2}$-Approach, EFRs can be modelled, however as the created models are non-executable and static, one model can only show one state of the system (e.g. one load state of the gear pair in Figure 1). In order to model changes of states in a system, the $C \& C^{2}$-Sequence Model has been developed (Albers et al., 2008). By linking individual $C \& C^{2}$-Models, it enables the documentation of EFRs through different states of the system. A state is defined as an arbitrarily long time span in which the amount and properties of the key elements of the $C \& C^{2}$-Approach can be seen as constant. A sequence describes a link of at least two states. As an example of a $\mathrm{C} \& \mathrm{C}^{2}$-Sequence Model, the interaction of a power tool and a screw is shown in Figure 2. On the left side, the overview over the sequence consisting of four states is shown. At the example of state 3 (centre) is shown how a state splits up in another four sub-states. On the right side, key elements in one of the sub-states are shown.
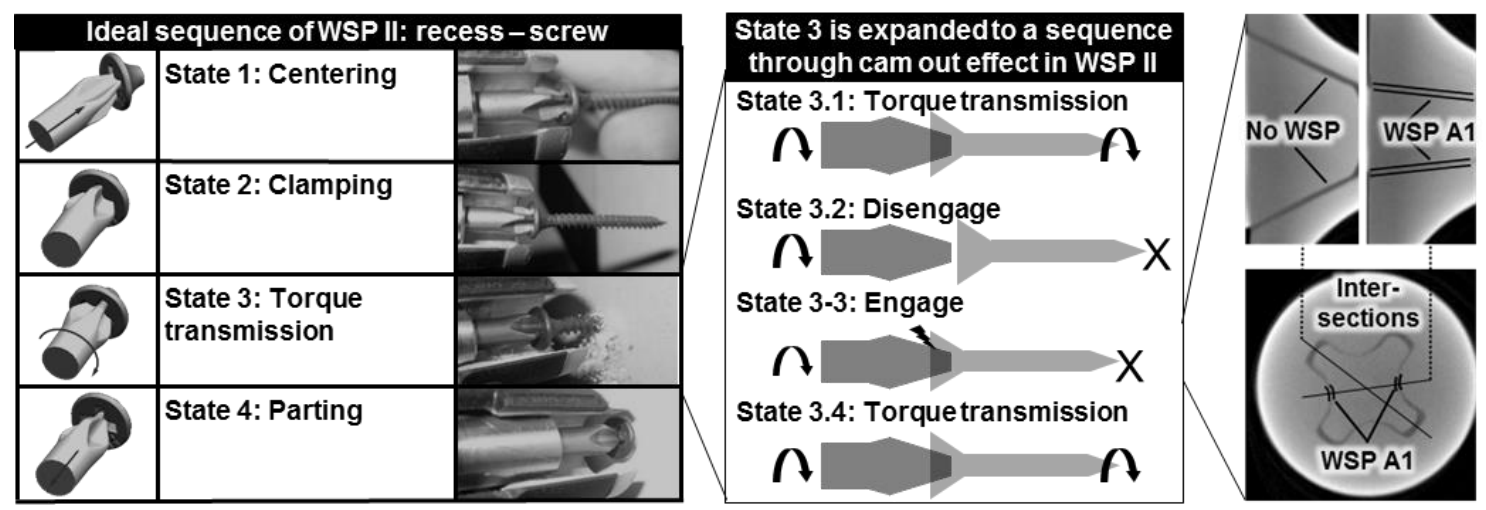

Figure 2: Section of a C\&C2-Sequence Model according to (Thau, 2013)

The $\mathrm{C} \& \mathrm{C}^{2}$-Sequence model is developed further into the comb-approach by Thau (2013). Its aim is to enable modelling of sequences in different parts of the system (locations of function fulfilment). However, the additional dimension makes it very difficult to use and no further usage of this approach is identified in literature.

\subsection{Modelling of logical states}

With the $C \& C^{2}$-Sequence Model, it is possible to differentiate system behaviour into states and investigate more complicated EFRs. However, there are systems that do not follow a fixed chronology of states, for example if a trigger influences the system behaviour. Here states can occur according to a logical sequence. In a development project in the field of power tools, the logical decomposition has been integrated into the $C \& C^{2}$-Sequence Model. Figure 3 shows a depiction of the logical states modelling. On the left, the overview-level shows a logical structure. Here logical dependencies and effects on the overall system can be represented, while the embodiment is not depicted. On the right, the detail-level shows one switch in two logical states. The key elements of the $\mathrm{C} \& \mathrm{C}^{2}$-Approach are modelled in a schematic sketch of the system. However, a combination of the modelling of logical states with the already existing dimensions of the $\mathrm{C} \& \mathrm{C}^{2}$-Sequence Model has not been found in literature. 


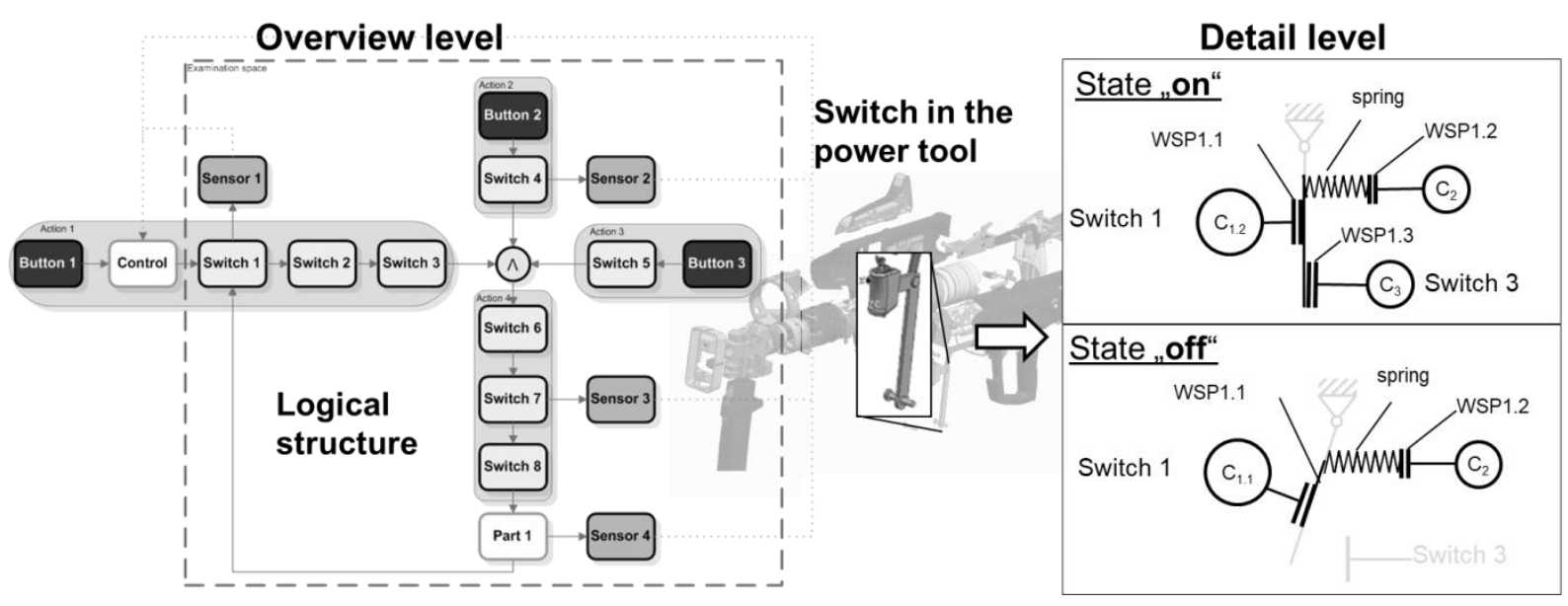

Figure 3: Example of a security switch in a power tool modelled with logical states in the C\&C²-Sequence Model according to (Matthiesen and Ruckpaul, 2012)

\section{THE STRUCTURE FOR THE C\&C ${ }^{2}$-SEQUENCE MODEL}

In this chapter, the identified dimensions of the developed structure for the $C \& C^{2}$-Sequence Model are described. Then an overview of the $C \& \mathrm{C}^{2}$-Sequence Model with this structure is shown and two academic examples clarify the possibilities of its usage in embodiment design. The implemented dimensions for the structure are based on the preliminary work described in chapter 2. Findings from the state of the art, like the dimensions of the SysML-diagrams are already covered by the different preliminary work, so the focus lays on developing the structure and ensure its applicability. The dimensions used for the structure are:

- The dimension logical state from section 2.3 is implemented as the superordinate dimension of the structure. It describes the possible system behaviour according to arbitrary internal or external triggers.

- The dimension state of the system is implemented as a dimension of the three-dimensional coordinate system that is built up in one logical state. This dimension is the central dimension of the preliminary $\mathrm{C} \& \mathrm{C}^{2}$-Sequence Model (see also section 2.2). It enables depiction of EFRs in technical systems that change their state during function fulfilment.

- The dimension level of detail is another dimension of the coordinate system. It is identified as a

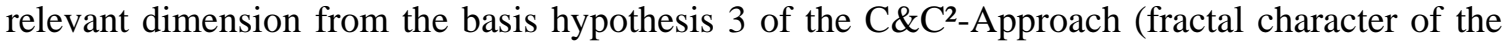
built model, see also section 2.1).

- The dimension location of function fulfilment is implemented as third dimension of the coordinate system. It is used when the need to adjust the system boundaries in modelling EFRs arises and is also described in section 2.2.

These dimensions are then connected in a way beneficial for design engineers by implementing a set of metadata for the single $\mathrm{C} \& \mathrm{C}^{2}$-Models. In this way their connection can be documented and communicated by design engineers.

In Figure 4, an overview of the structure for the $C \& C^{2}$-Sequence Model is depicted at the academic example of a gear box. The dimensions level of detail, location of the function fulfilment and state of the system are represented in a three-dimensional coordinate system that describes the systems behaviour in a certain logical state. The squares represent particular $C \& C^{2}$-Models in which the EFR is documented. The logical states as fourth dimension connect the coordinate systems. They are depicted as triggers that lead to different system behaviour according to their settings. 


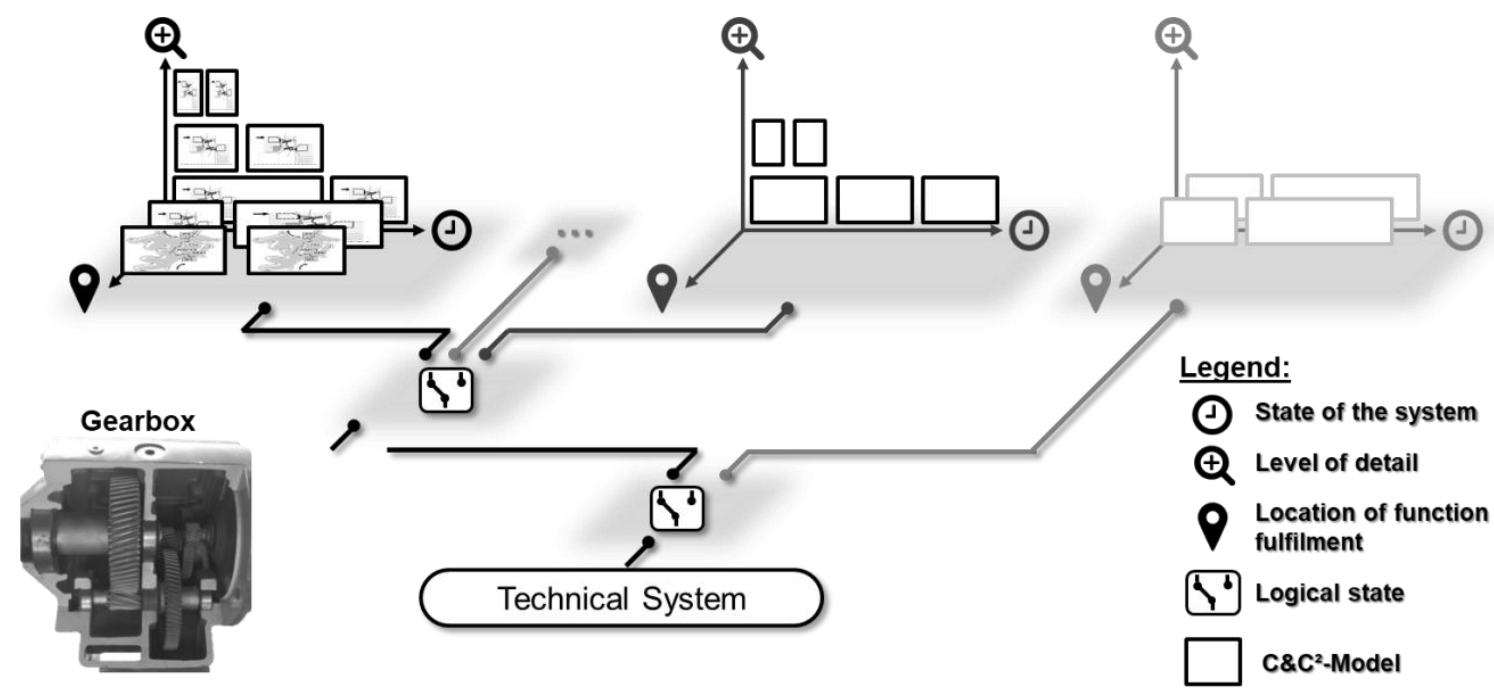

Figure 4: The structure for the C\&C²-Sequence Model - academic example

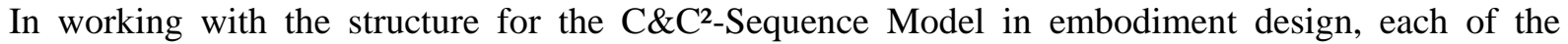
created $\mathrm{C} \& \mathrm{C}^{2}$-Model has a set of metadata that show where it belongs in the structure. From these metadata, different views can be filtered from the $\mathrm{C} \& \mathrm{C}^{2}$-Sequence Model. At the academic example of a gearbox, Figure 5 shows a derived view, where different states in the location of function fulfilment

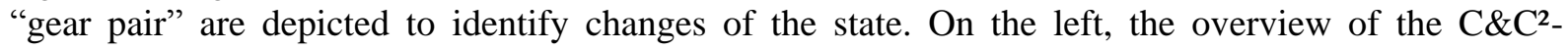
Sequence Model is shown. An intersection in the location of function fulfilment is shown, where the gear pair is located. It shows the $\mathrm{C} \& \mathrm{C}^{2}$-Models (Figure 5, right side), that describe how the force flow changes from WSP1.1 to WSP1.2 when the next gear tooth pair is engaged. The metadata are shown on top of the $C \& C^{2}$-Models using the icons from Figure 4. Here the counter for the state dimension is increased when the gear pair turns.

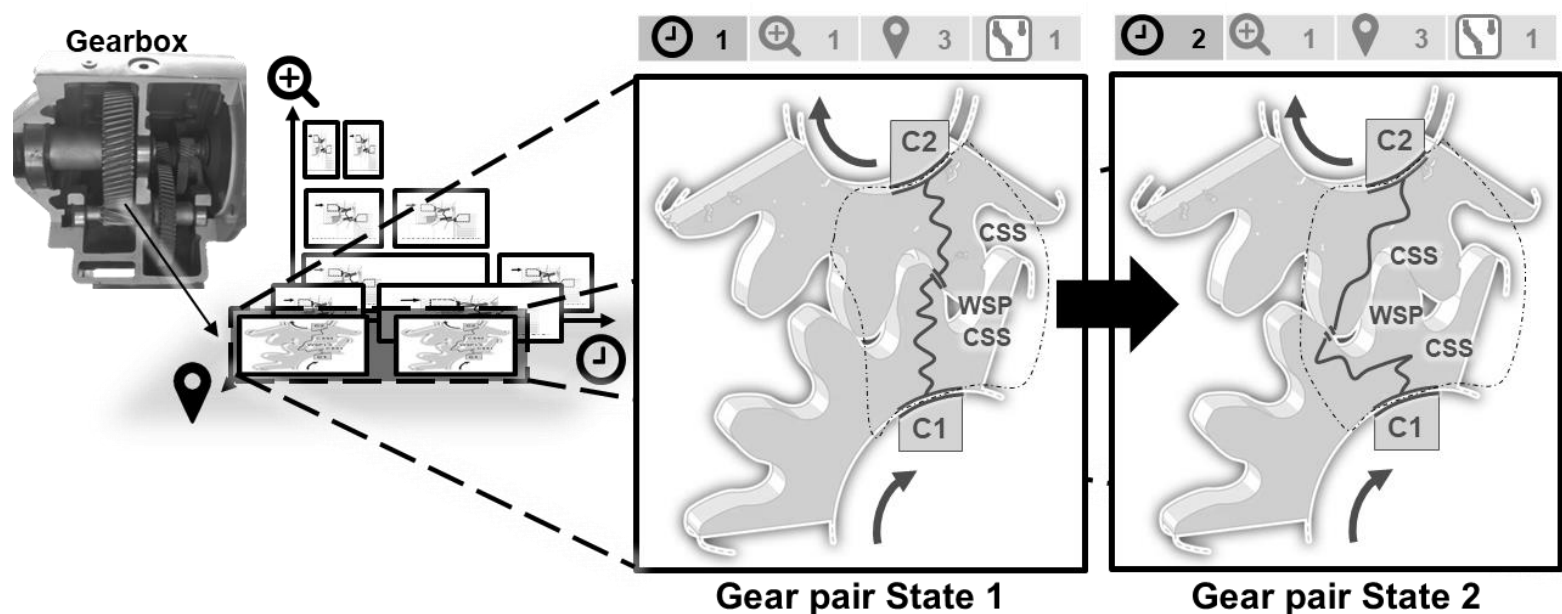

Figure 5: View of state changes from the academic C\&C2-Sequence Model of a gearbox

Another derived view of this $C \& C^{2}$-Sequence Model is shown in Figure 6, where different locations of function fulfilment are investigated in a certain state of the academic gearbox example. Here on the left the two bearings of the axis of one of the gears are shown as locations of function fulfilments. The intersection is inserted through the three locations of function fulfilment. On the right, the associated $\mathrm{C} \& \mathrm{C}^{2}$-Models of the gear pair and the bearings are shown. 


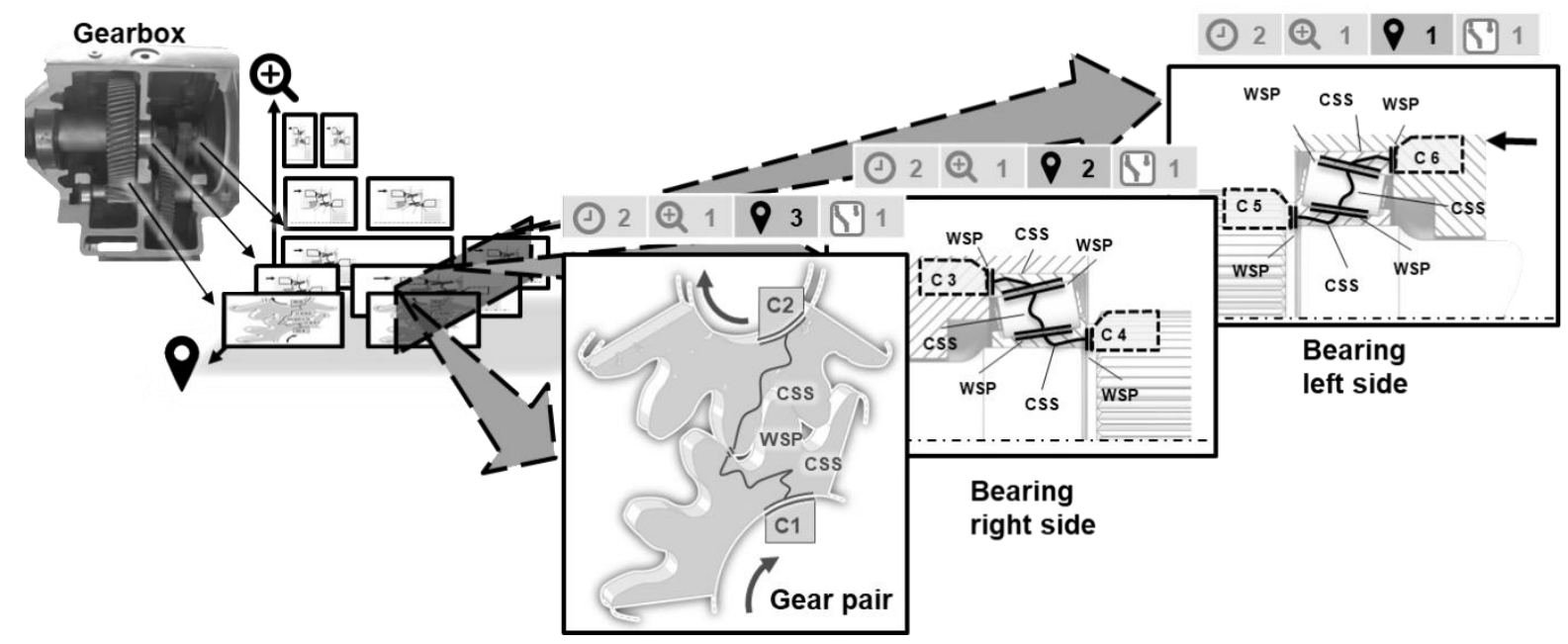

Figure 6: View of different locations of function fulfilment in one state from the academic C\&C2-Sequence Model of a gearbox

The aim of this structure is to support the investigation of function relevant parameters. With the

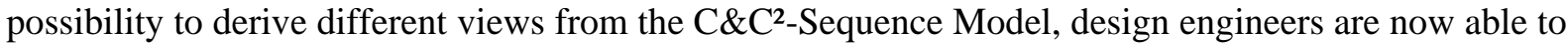
document their findings in a way that enables the reuse of gained knowledge and also simplifies communication.

\section{APPLICATION OF THE STRUCTURE FOR THE C\&.2 ${ }^{2}$-SEQUENCE MODEL}

The developed structure is applied in two development projects, where complicated systems had to be investigated and EFRs were difficult to gain, document and communicate.

\subsection{The sunroof system - application in an industrial case study}

With the developed modelling structure, an analysis project in corporate environment has been carried out. Aim was to investigate a sunroof system of a car with focus on identification of causes for unwanted system behaviour. For this, EFRs had to be identified, as it was unclear which embodiment parameters were responsible for the unwanted behaviour. In the analysis it became clear that the resulting $131 \mathrm{C} \& \mathrm{C}^{2}$-Models in this complicated system could not be structured in a manageable way with the preliminary $\mathrm{C} \& \mathrm{C}^{2}$-Sequence Model. With the developed structure it was possible to integrate the created $\mathrm{C} \& \mathrm{C}^{2}$-Models into the $\mathrm{C} \& \mathrm{C}^{2}$-Sequence Model and document the analysis results. An overview of the identified EFRs is shown in Figure 7. On the left, the regular operation sequence of the sunroof system is depicted. Here, 12 different locations of functional fulfilment are active in different states. Due to clarity, the models are shown in a schematic overview and the detailing dimension is only shown at the first location of function fulfilment. Here, the state at detail level zero (black beam in the back of the coordinate system) splits up into six states at detail level one. In one of these states a further detailing is necessary, which leads to another four states on detail level two.

Further logical states are modelled when the engine is off and external loads act on the sunroof. They are depicted in Figure 7 on the right. This results in four logical states in which different locations of function fulfilment are active and other parameters become relevant for the system behaviour than in the regular operation sequence. The identified EFRs were also integrated into the $\mathrm{C} \& \mathrm{C}^{2}$-Sequence Model and it was possible to derive different views to discuss the findings and use them for further analysis. 


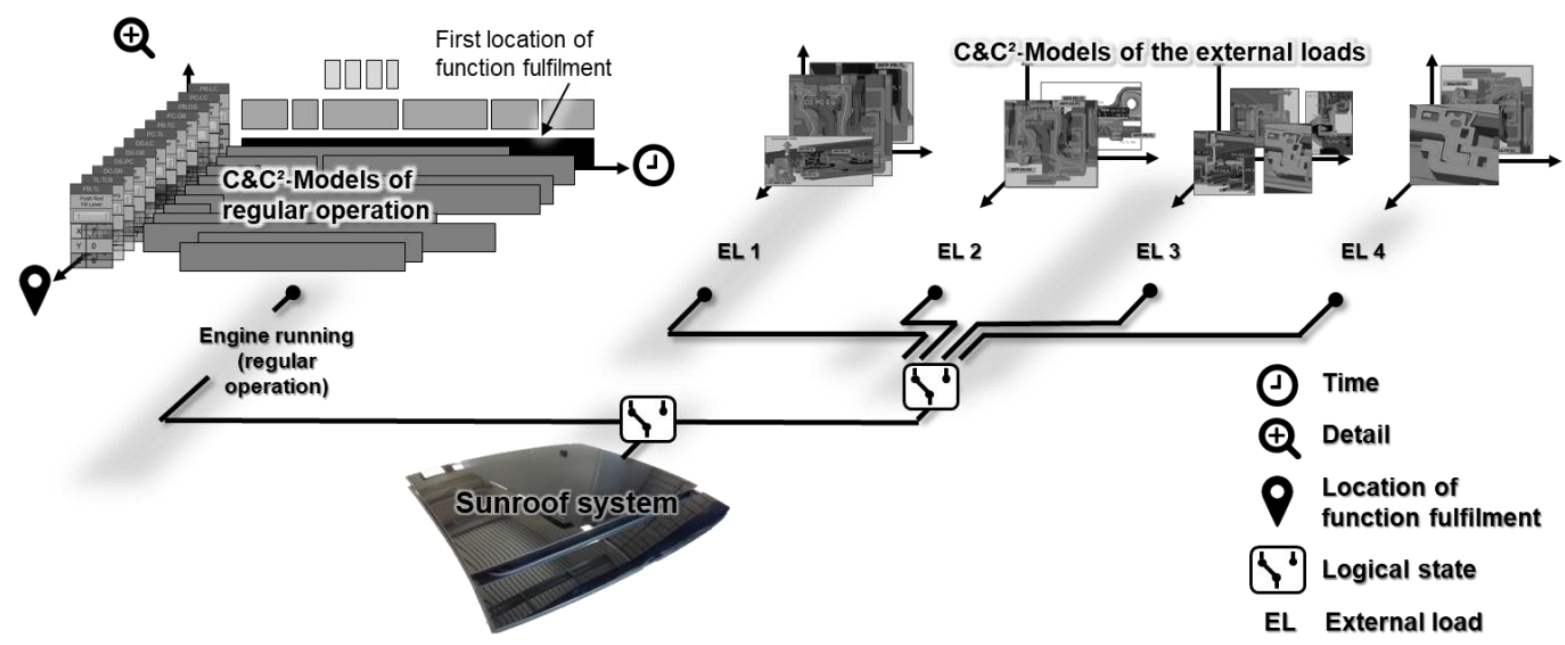

Figure 7: Overview of the $C \& C^{2}-$ Sequence Model of the sunroof system

An example of a finding of EFRs is shown in Figure 8. Here the force flow from the sunroof panel into the guide rail (linear sliding contact bearing) is shown in the $\mathrm{C} \& \mathrm{C}^{2}$-Models on the right and left. However, wear traces in the sled rail indicated an overload and a $\mathrm{C} \& \mathrm{C}^{2}$-Model was built up to investigate the cause for this (Figure 8, center). The neighbouring $C \& \mathrm{C}^{2}$-Models show, how the force generally is transmitted into the guide rail. Momentarily however, this force is conducted into the drive sled, which leads to wear of the sled rail. These findings were then integrated into the ${\mathrm{C} \& \mathrm{C}^{2}-}_{-}$ Sequence Model and the gained knowledge about EFRs was documented for discussion with the experts.

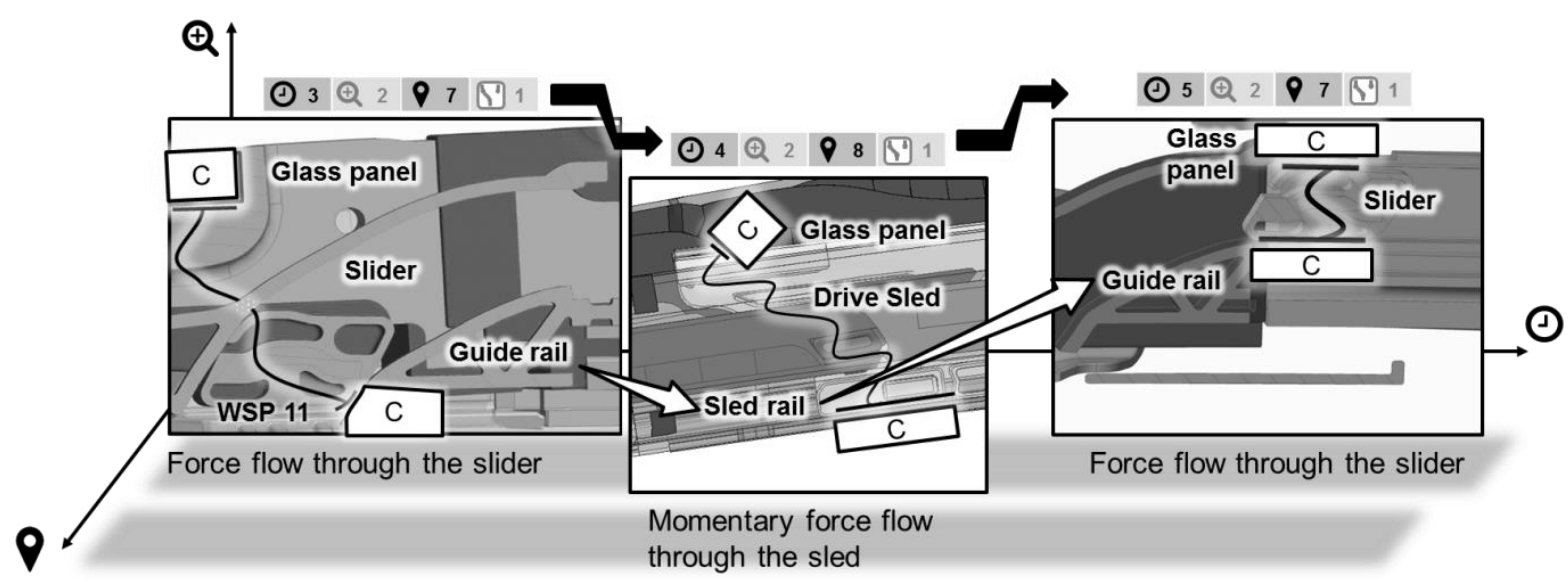

Figure 8: Change in the force flow through the sliders of the sunroof system

With this model it was possible to present gained insights about this very complicated system with the

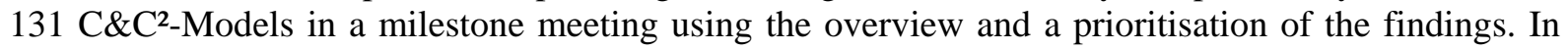
about $2 \mathrm{~h}$ the participating experts from different departments of the company could evaluate the findings, add their insights to the findings if necessary and decide on how to proceed on this basis. In this project, the structure for the $\mathrm{C} \& \mathrm{C}^{2}$-Sequence Model has shown potential in modelling and communicating EFRs in complicated systems.

\subsection{The clamping roller free wheel - application in an academic case study}

In a subsequently conducted academic development project, a double-locking clamping roller free wheel has been investigated. The aim of this project was to develop a fully functioning prototype that is manufacturable by using 2D rapid prototyping technology (laser cutting of high density fibre board and acrylic glass). For developing this prototype, the EFRs of the clamping roller free wheel had to be known and were investigated.

This clamping roller free wheel is a torque actuated clutch, which can transmit drive torque in both turning directions and block overload torque from the output shaft also in both directions. 
Requirements for the developed system are a high reliability of the function fulfilment, a quick exchange of system parts without special tools and the observability of the function fulfilment.

In an analysis phase of the systems development, $C \& C^{2}$-Models were created for each of its two main functions (transmit torque from drive and block torque from output shaft) and integrated into a $\mathrm{C} \& \mathrm{C}^{2}$ Sequence Model. The undefined position of the cylindrical roll at the beginning of the sequence was identified as a trigger which influences the following states. Figure 9 shows on the left the reference system and the developed prototype. Right from them, the created $C \& \mathrm{C}^{2}$-Sequence Model is shown.

The structure enable to estimate the influence of the change of embodiment parameters on its functions.

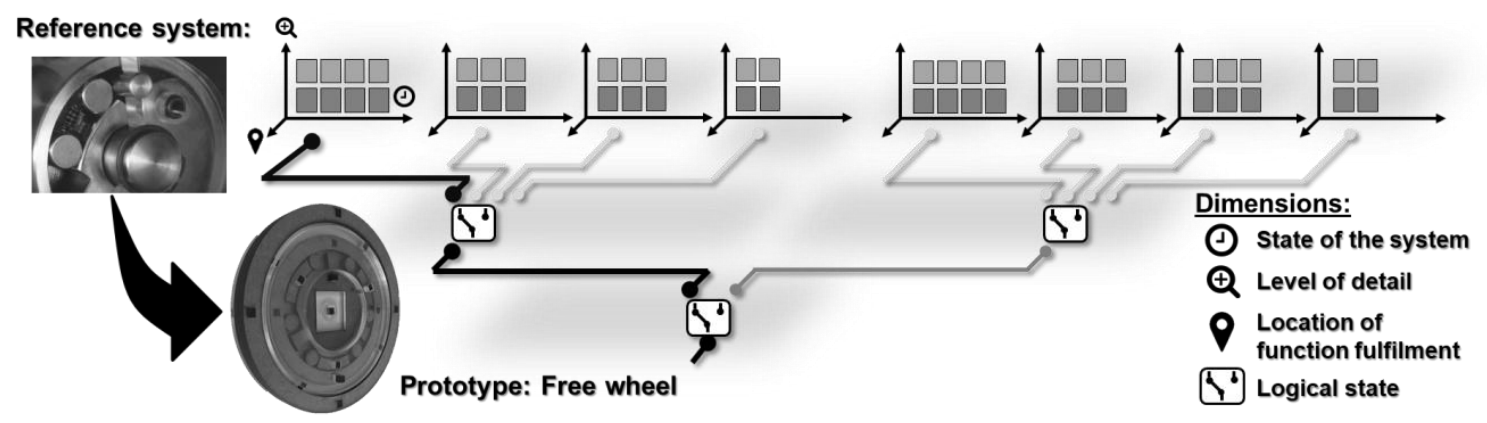

Figure 9: Structure of the C\&C2-Sequence Model of the clamping roller free wheel

As an example, in Figure 10 an intersection of the first logical state on the second level of detail, two states of the lower left roller were observed during function fulfilment.

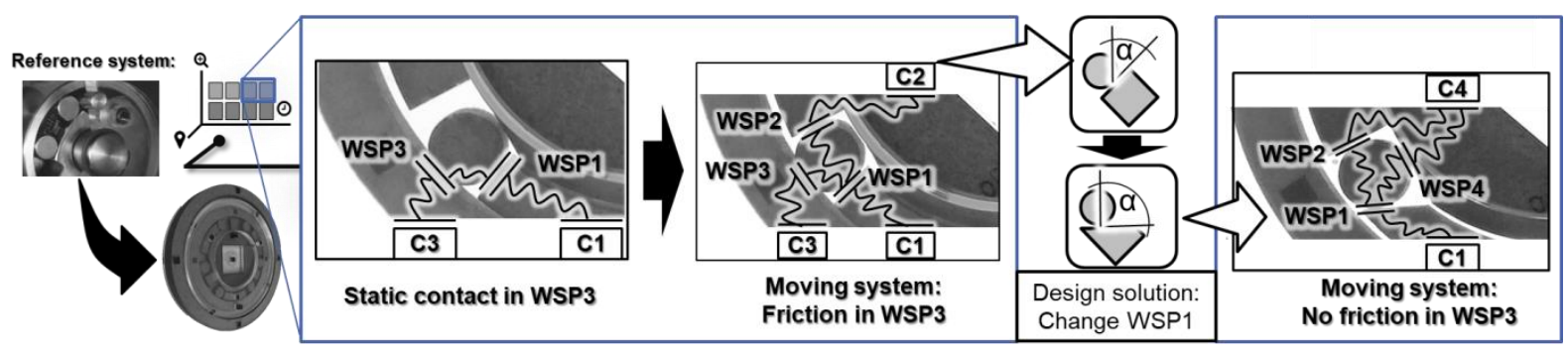

Figure 10: Unwanted friction loss in a state of the clamping roller free wheel and improved system

Here it is shown how the roller grinds the outer ring in WSP3 during force transmission between WSP1 and WSP2. It was assumed that WSP3 was a cause for low reliability of the function fulfilment through friction losses in the moving system. To verify this assumption, a design hypothesis of a function relevant parameter according to (Matthiesen et al., 2017) was derived and tested using the prototype shown in Figure 10 on the right. There, through change of a parameter in WSP1, WSP3 was prevented from occurring in this state. During the investigation, six design hypotheses were tested and their insights were implemented until the system fulfilled its requirements.

\section{DISCUSSION}

The developed structure for the $\mathrm{C} \& \mathrm{C}^{2}$-Sequence Model enabled its usage in modelling of two complicated systems. The four identified dimensions made it possible to integrate all built up models of EFRs into the structure, therefore the research question can be answered in the way that the dimensions logical state, detail, state of the system and location of function fulfilment can support the explication of the built up mental models of the design engineers in the analysis phase. It also showed that communication of findings is supported by using the structure. A limitation is, that in described development projects, the mechanical aspects were in focus. Dimensions which might be necessary to model for example electrical or hydraulic systems have not been investigated.

In the two development projects, design engineers with experience in model building of EFRs investigated the systems. It was shown that the structure was helpful to communicate insights about EFRs in a complicated system to design engineers with different fields of expertise. However, an investigation of the usability of the structure of the $C \& C^{2}$-Sequence Model for design engineers 
without experience in modelling EFRs is still necessary. The question, how this model can support

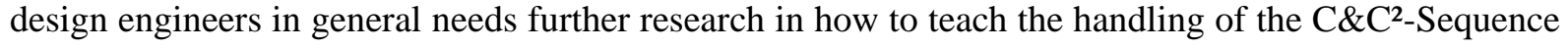
Model.

\section{CONCLUSION AND OUTLOOK}

In this contribution, a structure for the $\mathrm{C} \& \mathrm{C}^{2}$-Sequence Model has been developed to match the needs of design engineers to investigate embodiment function relations (EFRs) in complicated systems. This model has been used in two development projects. In the project in corporate environment, it was possible to integrate findings into the structure and document the gained knowledge about EFRs in a way that enabled other design engineers to understand them and extend the findings with their own knowledge. In the development project in academic environment, the structure was used to improve a system based on analysis of its EFRs. Here the documented insights were used to investigate, when which embodiment was participating in the function fulfilment. Concluding it can be said that the developed structure for the $\mathrm{C} \& \mathrm{C}^{2}$-Sequence Model can support design engineers in investigation of EFRs in complicated systems.

\section{REFERENCES}

Albers, A., Alink, T., Matthiesen, S. and Thau, S. (2008), "Support of System Analyses and Improvement in Industrial Design through the Contact \& Channel Model", in Proceedings of the 10th International Design Conference DESIGN 2008, Dubrovnik, Croatia, pp. 245-252.

Albers, A. and Wintergerst, E. (2014), “The Contact and Channel Approach (C\&C²-A): relating a system's physical structure to its functionality", in Chakrabarti, A. and Blessing, L.T.M. (Eds.), An Anthology of Theories and Models of Design: Philosophy, Approaches and Empirical Explorations, Springer, pp. 151-172.

Andreasen, M.M., Hansen, C.T. and Cash, P. (2015), Conceptual Design: Interpretations, Mindset and Models, Springer International Publishing, Cham, Switzerland.

Dorst, K. and Cross, N. (2001), "Creativity in the design process: co-evolution of problem-solution", Design Studies, Vol. 22 No. 5, pp. 425-437.

Ehrlenspiel, K. and Meerkamm, H. (2017), Integrierte Produktentwicklung: Denkabläufe, Methodeneinsatz, Zusammenarbeit, 6., vollständig überarbeitete und erweiterte Auflage, Carl Hanser Verlag GmbH \& Co. KG, München, Wien.

Gero, J.S. and Kannengiesser, U. (2004), “The situated function-behaviour-structure framework”, Design Studies, Vol. 25 No. 4, pp. 373-391.

Matthiesen, S. (2002), "Ein Beitrag zur Basisdefinition des Elementmodells "Wirkflächenpaare \& Leitstützstrukturen” zum Zusammenhang von Funktion und Gestalt technischer Systeme”, Dissertation, Forschungsberichte des Instituts für Maschinenkonstruktionslehre und Kraftfahrzeugbau, Universität Karlsruhe (TH), Karlsruhe, Germany, 2002.

Matthiesen, S. (2011), "Seven Years of Product Development in Industry - Experiences and Requirements for Supporting Engineering Design with 'Thinking Tools"', in Proceedings of the 18th International Conference on Engineering Design. ICED’11. Copenhagen, Denmark, pp. 236-245.

Matthiesen, S., Grauberger, P., Sturm, C. and Steck, M. (2018), "From Reality to Simulation - Using the C\&C²Approach to Support the Modelling of a Dynamic System", in Procedia CIRP, Elsevier B.V.

Matthiesen, S. and Ruckpaul, A. (2012), "New Insights on the Contact\&Channel-Approach - Modelling of Systems with Several Logical States”. In Proceedings of the International Design Conference DESIGN 2012. Dubrovnik, Croatia, pp. 1019-1028

Morkos, B., Shankar, P. and Summers, J.D. (2012), "Predicting requirement change propagation, using higher order design structure matrices: an industry case study”, Journal of Engineering Design, Vol. 23 No. 12, pp. 905-926.

Thau, S. (2013), "Heuristiken zur Analyse und Synthese technischer Systeme mit dem C\&C2-Ansatz auf Basis von Entwicklungsprojekten im industriellen Umfeld", Dissertation, Forschungsberichte des IPEK - Institut für Produktentwicklung, Karlsruher Institut für Technologie, Karlsruhe, Germany, 2013.

Weber, C. (2014), "Modelling Products and Product Development Based on Characteristics and Properties", in Chakrabarti, A. and Blessing, L.T.M. (Eds.), An Anthology of Theories and Models of Design: Philosophy, Approaches and Empirical Explorations, Springer, pp. 327-352.

Weilkiens, T. (2007), Systems engineering with SysML/UML: Modeling, analysis, design, The OMG press, Morgan Kaufmann OMG Press/Elsevier, Amsterdam, Boston. 